\title{
Inventaire rapide des principaux entomophages de la cochenille du manioc Phenacoccus manihoti (Homoptera : Pseudococcidae) en République du Congo
}

\author{
Valentin DIBANGOU ${ }^{1 *}$, Mireille BELLE MBOU ${ }^{1,2}$, Nazaire LOUBAKI NTOLO ${ }^{1}$, et \\ Grâce NIANGA-BIKOUTA ${ }^{1}$ \\ ${ }^{1}$ Laboratoire de Biodiversité et Ecologie Animales, Faculté des Sciences et Techniques, Université Marien \\ Ngouabi, BP 69, Brazzaville, République du Congo. \\ ${ }^{2}$ Rectorat de Limoges, 13 Rue Francois Chénieux, 87000 Limoges, France. \\ *Auteur correspondant, E-mail : dibangou@hotmail.com; Tel : +242-05-586-54-34
}

\section{RESUME}

Dès l'année 1970, une baisse de production en tubercules de manioc de l'espèce Manihot esculenta Crantz (Euphorbiacée) a été constatée en Afrique. L'un des principaux ravageurs de cette plante est la cochenille farineuse du manioc. Au Congo, la lutte contre celui-ci s'est organisée dès 1982 par l'introduction d'un parasitoïde hyménoptère, Epidinocarcis lopezi. Mais cet insecte auxiliaire s'est très vite retrouvé à son tour la proie d'hyperparasites qui freinent ainsi son expansion et limitent son efficacité. La présente étude fait un inventaire des entomophages de la cochenille du manioc Phenacoccus manihoti. Elle s'est déroulée dans trois sites retenus dans la ville de Brazzaville : Kombé, jardin d'essai et Lycée Savorgnan de Brazza (LSB). La récolte des momies a été effectuée sur des feuilles infestées, les arthropodes ayant émergé ont été collectés et identifiés, les taux d'émergence et d'hyperparasitisme ont été déterminés. Les résultats obtenus montrent un taux d'hyperparasitisme élevé (60 et 66,66\%) et une faible abondance d'E. lopezi comparé aux hyperparasites (22 hyperparasites pour 13 parasitoïdes). Un autre parasitoïde Acerophagus coccois Smith non encore décrit jusqu'ici au Congo a été découvert. Il pourrait avoir été introduit accidentellement avec les boutures importées et expérimentées dans le but d'améliorer les performances du manioc.

(C) 2020 International Formulae Group. All rights reserved.

Mots clés : Lutte biologique, Auxiliaire, Entomocénose, Momie, Brazzaville, Congo.

\section{Rapid inventory of the main entomophagous species of the cassava mealybug Phenacoccus manihoti (Homoptera: Pseudococcidae) in the Republic of Congo}

\section{ABSTRACT}

As early as 1970, a decrease in the production of cassava tubers of the species Manihot esculenta Crantz (Euphorbiaceae) was observed in Africa. One of the main pests of this plant is the cassava mealybug. In the Congo, the fight against it was organized in 1982 with the introduction of a hymenoptera parasitoid, Epidinocarcis lopezi. But this auxiliary insect very quickly found itself the prey of hyperparasites, which thus 
slowed down its expansion and limited effectiveness. The present study makes an inventory of the entomophages of the cassava mealybug Phenacoccus manihoti. It took place in three selected sites in the city of Brazzaville: Kombé, test garden and Lycée Savorgnan de Brazza (LSB). The mummies were harvested from infested leaves, emerging arthropods were collected and identified, rates of emergence and hyperparasitism were determined. The results obtained reached a high rate of hyperparasitism (60 and 66.66\%) and a low abundance of E. lopezi compared to hyperparasites (22 hyperparasites for 13 parasitoids). Another parasitoid Acerophagus coccois Smith not yet described in Congo has been discovered. It could have been created accidentally with the imported cuttings and tested in corn to improve cassava performance.

(C) 2020 International Formulae Group. All rights reserved.

Keywords: Biological pest control, Auxiliary, Entomocenosis, mummy, Brazzaville, Congo.

\section{INTRODUCTION}

En Afrique, les insectes ravageurs touchent depuis plus d'une décennie l'une des plus importantes cultures vivrières, le manioc Manihot esculenta Crantz (Euphorbiacée), qui est une plante de base, supplantant dans certaines régions les plantes locales telles que l'igname, la patate douce, la banane plantain et le sorgho (Pynaert, 1951; Daouda, 2018). La cochenille farineuse du manioc Phenacoccus manihoti Matile-Ferrero (Homoptera, Pseudococcidae) signalée au Congo en 1973 (Silvestre, 1973; Yonow \& al., 2017) constitue l'un des principaux ravageurs (Abderahim \& al 2018).

La lutte biologique a très vite été préconisée comme un moyen efficace pour lutter contre les cochenilles (Neuenschwander, 2001). En Afrique et plus particulièrement au Congo, $P$. manihoti est attaquée par la guilde habituelle de prédateurs polyphages ou oligophages dont les plus importants sont les coccinellidés telles que, Hyperaspis spp., Exochomus sp, et Diomus sp. Ces dernières se rencontrent généralement à de fortes densités au niveau de la cochenille du manioc (Obame Minko \& Békon, 2005). Sont également utilisés dans la lutte biologique, des champignons entomopathogènes qui attaquent et détruisent naturellement et de manière ciblée par des maladies fongiques les insectes ravageurs (Butt, 2002). C'est le cas de Fusarium sp qui provoque un taux de mortalité avoisinant les $100 \%$ des adultes du criquet Brachytrupes megacephalus (Lakhdari \& al., 2015). Il est à noter que $P$. manihoti est aussi attaqué par des parasitoïdes (Ben-Dov \& German, 2003). Les parasitoïdes sont des organismes dont les larves se développent aux dépens d'un seul hôte (Boivin, 2001).

Malgré la rapide adaptation de près d'une centaine d'espèces entomophages à l'égard de la cochenille du manioc (Obame Minko \& Békon, 2005), leur action régulatrice semble faible.

Cependant, il existe un complexe d'ennemis naturels potentiellement utilisables dans la lutte biologique contre la Cochenille du manioc en Afrique (Atachi \& al, 2011). Parmi ceux-ci la présence d'un entomophage bien connu, Epidinocarsis (Apoanagyrus) lopezi (De Santis), un Hyménoptère Encyrtidae parasitoïde de la Cochenille du manioc s'est révélée plus prometteuse (Herren \& Lema, 1982 ; Lema \& Herren, 1985). C'est ainsi qu'E. lopezi a été introduit au Congo en 1982 par «international institute of tropical agriculture, (2000 IITA)». De nombreux travaux ont alors été entrepris en vue de connaitre la biologie, la physiologie de la reproduction et le type de parasitisme développé par cet auxiliaire (Biassangama, 1984 ; Nenon \& al., 1985 ; Odeyibi \& Bokonon-Ganta, 1986, Iziquel \& Le Rü, 1989), afin d'apprécier son impact réel et son aptitude à assurer la régulation des populations de P. manihoti.

Au Congo et au Sénégal, malgré le succès de l'acclimatation du parasitoïde, des pullulations du ravageur ont persisté (Neuenschwander, 2001). L'une des causes possibles de son manque d'efficacité réside dans l'existence de ce complexe hyperparasitaire très riche infligeant à l'auxiliaire une mortalité considérable (Le Rü, 1989). Dans le cas spécifique du Congo, 7 espèces d'Hyménoptères hyperparasites, imposent à E. lopezi près de $70 \%$ de mortalité 
(Biassangama \& Moussa, 1987 ; Iziquel \& Le Rü, 1989). Ainsi ce complexe des hyperparasites qui s'est structuré et remarquablement diversifié en quelques années seulement autour d'E. lopezi pose un problème d'efficacité dans la lutte biologique contre $P$. manihoti. Trente-six ans après l'introduction d'E. lopezi, la présente étude est effectuée dans le but d'évaluer le comportement actuel de cet auxiliaire notamment sur sa capacité à réduire significativement les populations du ravageur.

\section{MATERIEL ET METHODES}

\section{- Présentation du milieu d'étude}

L'étude s'est déroulée pendant 8 semaines dans le département autonome de Brazzaville au cours des mois de juillet et août de l'année 2018 (saison sèche), période où les effectifs de cochenille sont les plus importants. Brazzaville est située à $-4^{\circ} 15^{\prime} 56$ de latitude Sud et $15^{\circ} 16^{\prime} 59$ de longitude Ouest. Elle est située à 320 mètres en moyenne au-dessus du niveau de la mer, le long du fleuve Congo.

La température moyenne annuelle est de $25.3{ }^{\circ} \mathrm{C}$ et les précipitations sont en moyenne de $1273.9 \mathrm{~mm}$ d'eau. L'humidité relative se situe souvent autour de la saturation ; elle est comprise entre 95 et $100 \%$ H.R. de jour comme de nuit. L'effet des hautes températures des heures chaudes de la journée fait tomber ces valeurs de 60 à $65 \%$ H.R. mais rarement en-dessous.

Cette situation géographique et ces facteurs climatiques, sont propices au développement des plantations de manioc dans des zones périurbaines.

Les échantillonnages sont effectués dans des champs de manioc de 2 ans environ. C'est en effet à cet âge que l'abondance des cochenilles y est plus grande (Fabre \& Le Rü, 1988). Trois sites ont été choisis. Il s'agit des sites de Kombé, du Jardin d'essai et du lycée Savorgnan de Brazza (LSB).

\section{Le site de Kombé}

Le centre Agri Congo de Kombé est implanté dans la zone sud de la ville de Brazzaville, dans l'arrondissement $\mathrm{n}^{\circ} \quad 8$ Madibou. Il occupe une superficie de quinze (15) hectares. La récolte des échantillons s'est effectuée sur une superficie d'environ 4 ha.

\section{Le site du Lycée Savorgnan de Brazza}

Les parcelles de culture de manioc de ce site, sont situées dans l'enceinte du Lycée Savorgnan de Brazza et mesurent environ une superficie de 2 ha. Les échantillonnages ont été effectués sur l'ensemble de cette superficie. Le Lycée Savorgnan de Brazza est situé dans l'arrondissement 2 Bacongo.

\section{- $\quad$ Le site du jardin d'essai}

Le jardin d'essai est situé proche du campus I affecté aux étudiants, le long de la rivière «maladie du sommeil» situé dans l'arrondissement1, Makélékélé. Des parcelles de culture de manioc y sont disséminées et occupent environ une superficie d'environ 2 ha. Les échantillonnages effectués ont concerné l'ensemble de cette superficie.

\section{METHODES}

\section{- Echantillonnage du parasitoüde $E$.} lopezi et de ses hyperparasites : L'échantillonnage du parasitoïde et des hyperparasites associés s'est effectué à partir des momies recueillies sur les feuilles de manioc infestées et récoltées dans les champs au niveau des 3 sites indiqués ci-dessus. Au laboratoire, les momies ont été délicatement détachées des feuilles à l'aide des pinceaux et aiguilles sous une loupe binoculaire de marque Leica EZ4 D. Elles ont ensuite été placées dans des pots en plastique mesurant $8 \mathrm{~cm}$ de diamètre sur $7 \mathrm{~cm}$ de profondeur et fermées avec une toile aux mailles très fines de $0,1 \mathrm{~mm}$ de diamètre et conservées à température ambiante au laboratoire. Certaines d'entre elles, ont éclot au bout de 24 heures tandis que d'autres encore immatures l'ont fait progressivement pendant 2 semaines au maximum.

Afin de récupérer les individus ayant émergé des momies, le pot est placé sous la loupe binoculaire. A l'aide d'un petit pinceau la toile est humectée avec de l'alcool à $70^{\circ}$ de façon à empêcher les arthropodes qui s'y agrippent de s'échapper à l'ouverture du pot. La toile et le fond du pot sont méticuleusement inspectés afin 
de récupérer les insectes à l'aide des petits pinceaux et le bout des grosses aiguilles. Ces derniers sont conservés dans de l'alcool à $70^{\circ}$ pour être ultérieurement identifiés et photographiés. Les pots sont refermés. Selon les stades de développement et d'arrivée au laboratoire, d'autres émergences ont été observées après éclosion des momies les jours suivants.

Les images des échantillons ont été prises à l'aide d'une caméra Digital Sight DS-U3 de marque Nikon, couplée à une loupe SMZ1000 de la même marque dont le Grossissement est de $4 \mathrm{x}$ à 480x (plage de zoom : 0.8x à 8x + oculaire : $\mathrm{C}$ W 10x). Lesdites images sont ensuite transférées à un $\mathrm{PC}$ et traitées avec le logiciel NIS-Elément de Nikon (Echelle, mesure, annotations).

La détermination taxonomique a été réalisée à partir des images et à l'aide de la clé d'identification de Atachi et al., (2011). Elle a ensuite été soumise à l'expertise de la liste de diffusion bioinsect du groupe RENATER (Réseau National de Télécommunications, l'Enseignement et la Recherche) pour confirmation basé à Montpellier en France.

\section{- Détermination du taux d'émergence (TE) \\ des individus au sein des momies}

Ce taux correspond au nombre d'insectes qui sortent des momies par rapport au nombre de momies:

$$
\mathrm{TE}=\frac{\text { nombre } d^{\prime} \text { individus ayant emergé }}{\text { nombre de momies }} \times 100
$$

\section{- Détermination du taux d'hyperparasitisme (TH)}

L'hyperparasitisme est une valeur qui donne une indication sur l'activité des hyperparasites responsables de la mortalité des parasitoïdes primaires. Il reflète la nature et l'importance des interactions interspécifiques existantes.

Le taux d'hyperparasitisme correspond au rapport entre le nombre d'hyperparasites émergés des momies sur le nombre moyen d'imagos obtenus en totalité (E. lopezi + hyperparasites), (Iziquel Y. \& Le Rü, 1989).

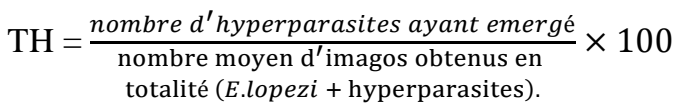

RESULTATS

- Nombre de momies et d'arthropodes obtenus, taux d'émergence

Un total de 275 momies a été récolté sur l'ensemble des 3 sites. De ces momies, 135 arthropodes ont émergé. Le nombre de momies par site, le nombre d'arthropodes ainsi que les taux d'émergence sont indiqués dans le Tableau 1.

Le taux d'émergence calculé à l'échelle des 3 sites revient à $49.09 \%$. Kombé est le site qui a le taux d'émergence le plus élevé.

\section{Identification, abondance et statut des} arthropodes émergés des momies.

Cinq espèces d'arthropodes dont le rôle est indiqué dans le Tableau 2 et appartenant tous à l'ordre des hyménoptères ont été identifiées. Il s'agit de :

- Acerophagus coccois Smith (Figures 1a et $1 \mathrm{~b}$. Numériquement, il est apparu plus abondant qu'E. lopezi (Tableau 2).

- Apoanagyrus (=Epidinocarcis) lopezi De santis, 1964 (Hymenoptera Encyrtidae) (Figure 2)

- Prochiloneurus insolitus (Alam, 1961) (Hymenoptera Encyrtidae), (Figure 3)

- Chartocerus hyalipennis (Hayat, 1970) (Hymenoptera Signiphoridae) (Figure 4)

- Pachineuron sp (Thomson, 1876) (Hymenoptera Aphelinidae) (Figure 5)

\section{Taux d'hyperparasitisme}

Les taux d'hyperparasitisme de chacun des 3 sites sont représentés dans le Tableau 3. Tous les hyperparasites identifiés sont solitaires, chaque individu provenant d'une émergence correspond à une momie à l'exception de Chartocerus hyalipennis Hayat qui est une espèce grégaire. Son effectif est néanmoins pris en compte dans le calcul. 
Tableau 1 : Nombre de momies et d'arthropodes obtenus dans les trois sites investigués

\begin{tabular}{lcccc} 
& Jardin d'essai & LSB & Kombé & Total \\
\hline Nombre de momies récoltées & 74 & 74 & 127 & 275 \\
\hline Nombre d'arthropodes émergés & 28 & 31 & 76 & 135 \\
\hline Taux d'émergence (\%) & 37,83 & 41,89 & 59,84 \\
\hline
\end{tabular}

Tableau 2 : Qualité et abondance des parasitoïdes et hyperparasitoïdes obtenus dans les 3 sites

\begin{tabular}{|c|c|c|c|c|c|c|c|}
\hline \multirow[b]{2}{*}{ Ordre } & \multirow[b]{2}{*}{ Familles } & \multirow[b]{2}{*}{ Genres et espèces } & \multirow[b]{2}{*}{ Statut } & \multicolumn{3}{|c|}{ Abondance dans chaque site } & \multirow[b]{2}{*}{ Total } \\
\hline & & & & $\begin{array}{l}\text { Jardin } \\
\text { d'essai }\end{array}$ & LSB & Kombé & \\
\hline \multirow{6}{*}{ 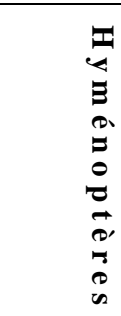 } & Encyrtidae & Epidinocarcis lopezi & Parasitoïde & 4 & 4 & 5 & 13 \\
\hline & Encyrtidae & Prochiloneurus insolitus & Hyperparasite & 1 & 3 & 2 & 6 \\
\hline & Encyrtidae & Acerophagus coccois & Parasitoïde & 18 & 21 & 61 & 100 \\
\hline & Signiphoridae & Chartocerus hyalipennis & Hyperparasite & 3 & 1 & 5 & 9 \\
\hline & Aphelinidae & Pachineuron $s p$ & Hyperparasite & 2 & 2 & 3 & 7 \\
\hline & & Total & & 28 & 31 & 76 & 135 \\
\hline
\end{tabular}

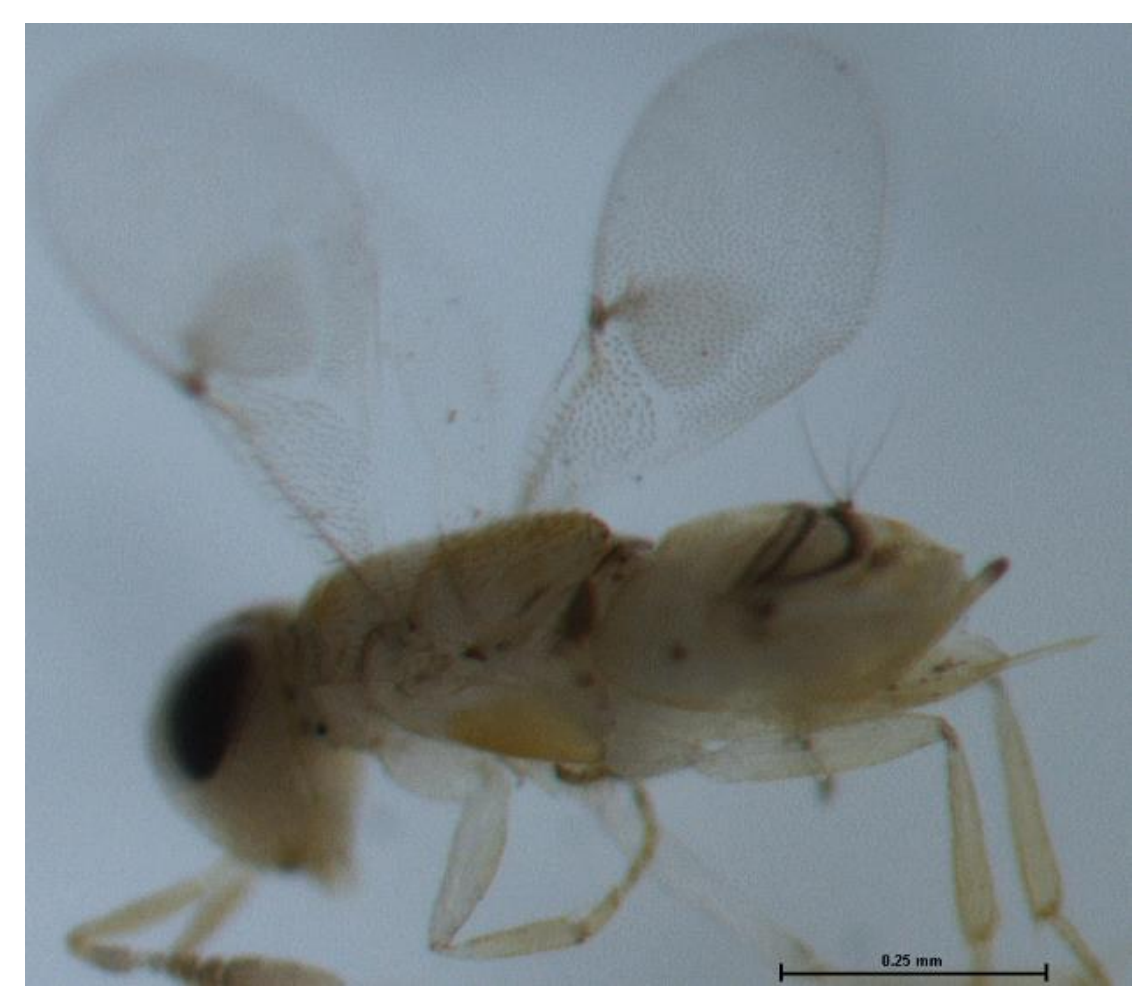

Figure 1a : Acerophagus coccois (vue latérale). 


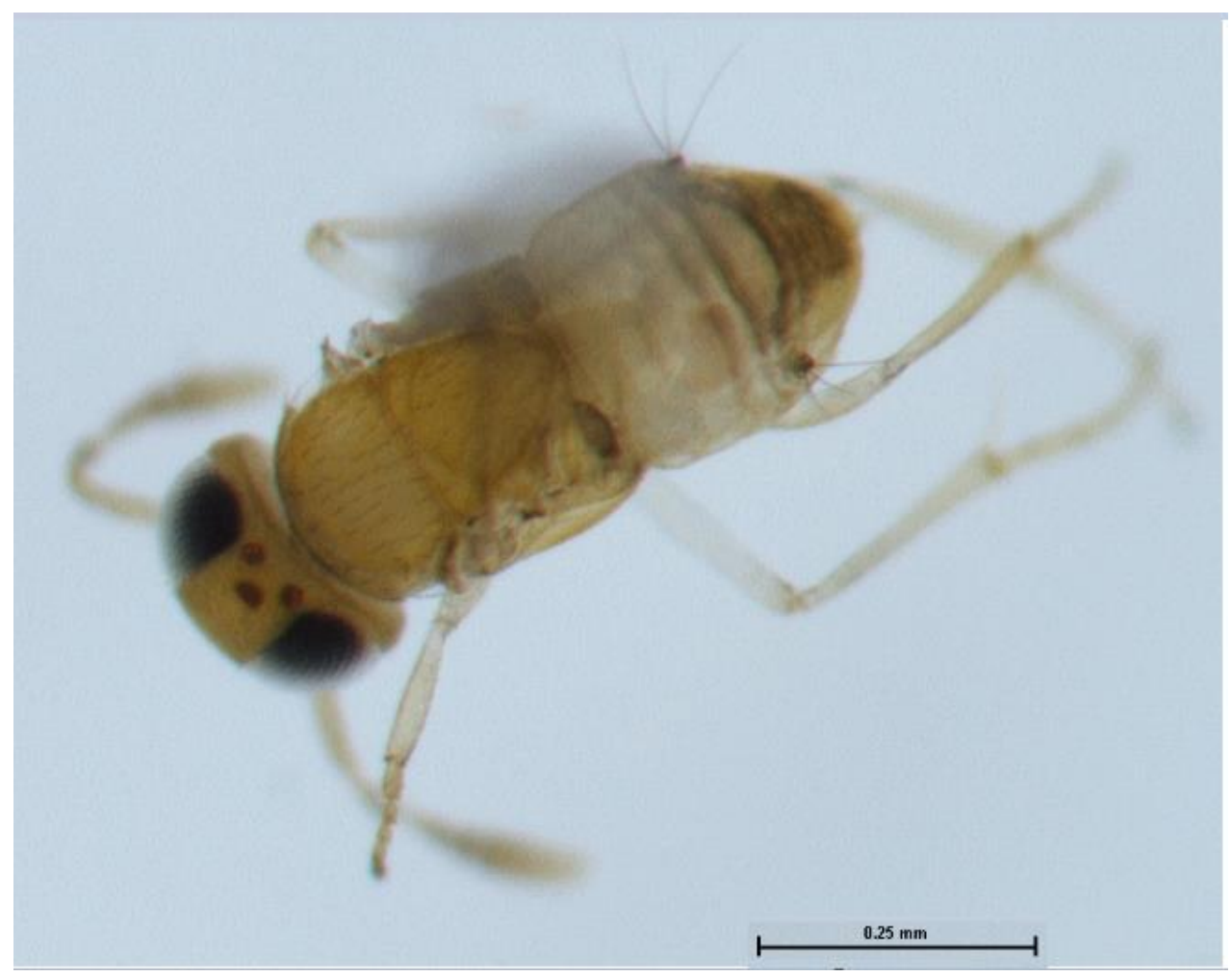

Figure 1b : Acerophagus coccois (vue dorsale).

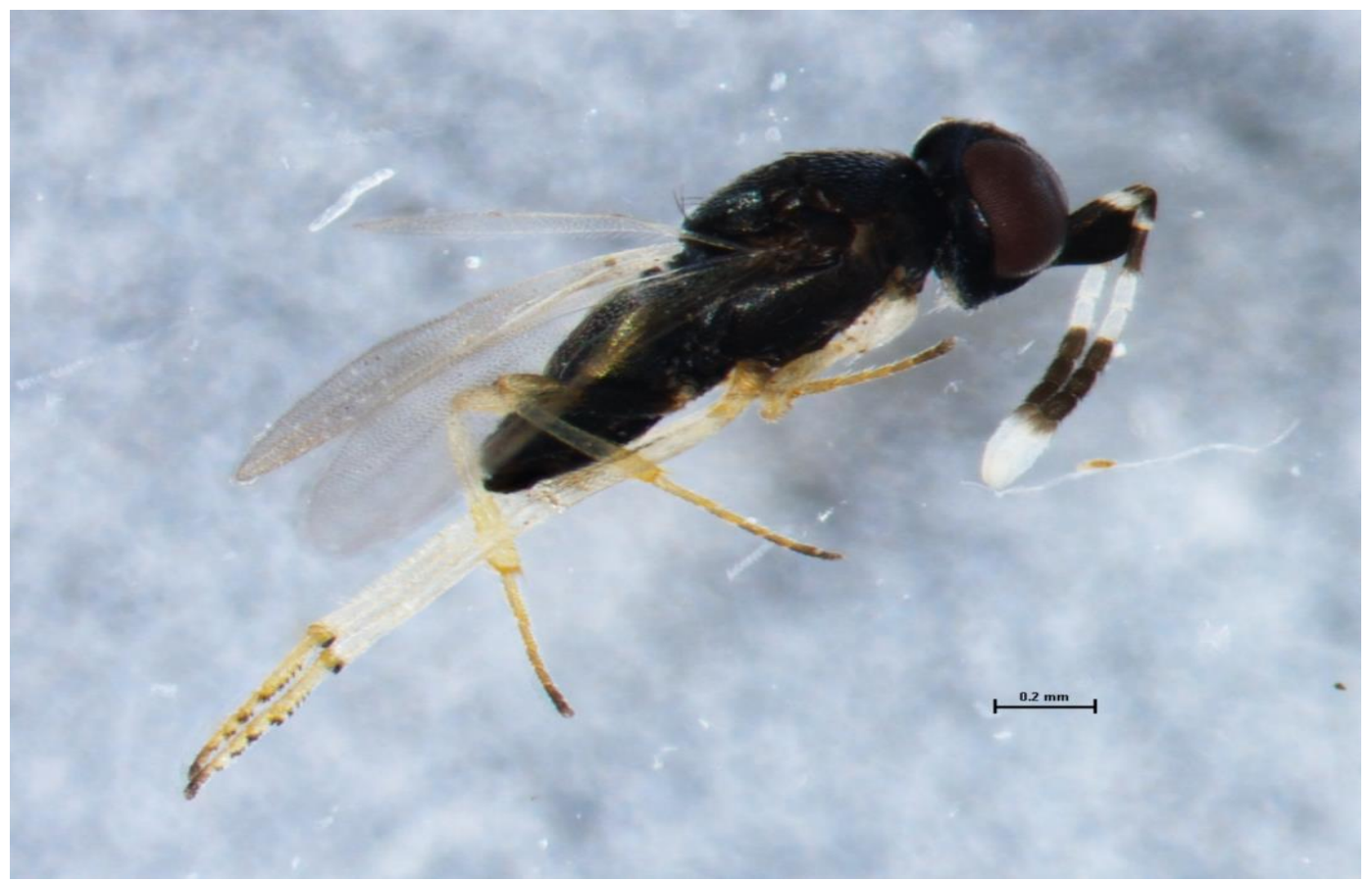

Figure 2 : Epidinocarcis lopezi. 
V. DIBANGOU et al. / Int. J. Biol. Chem. Sci. 14(7): 2517-2527, 2020

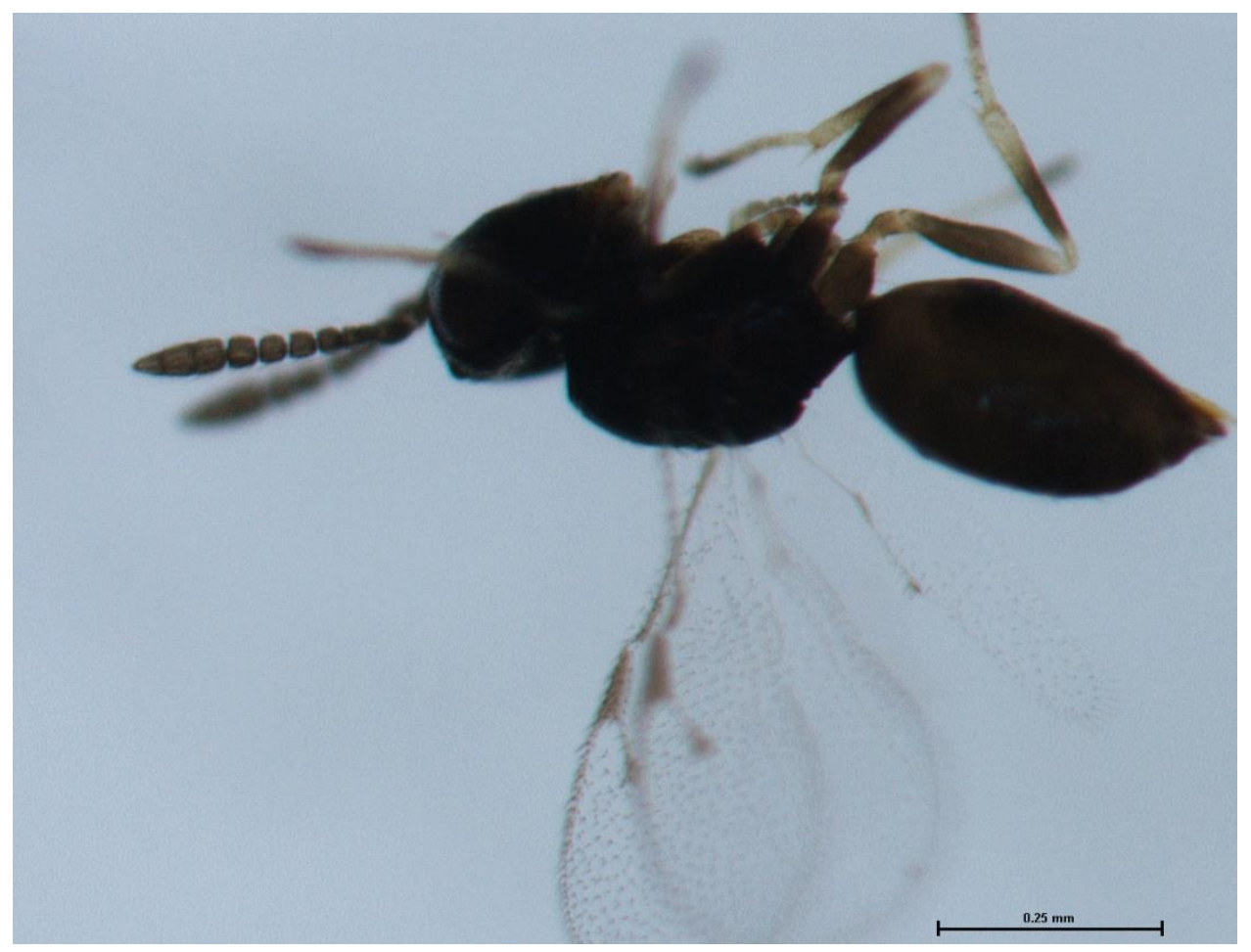

Figure 3 : Prochiloneurus insolitus.

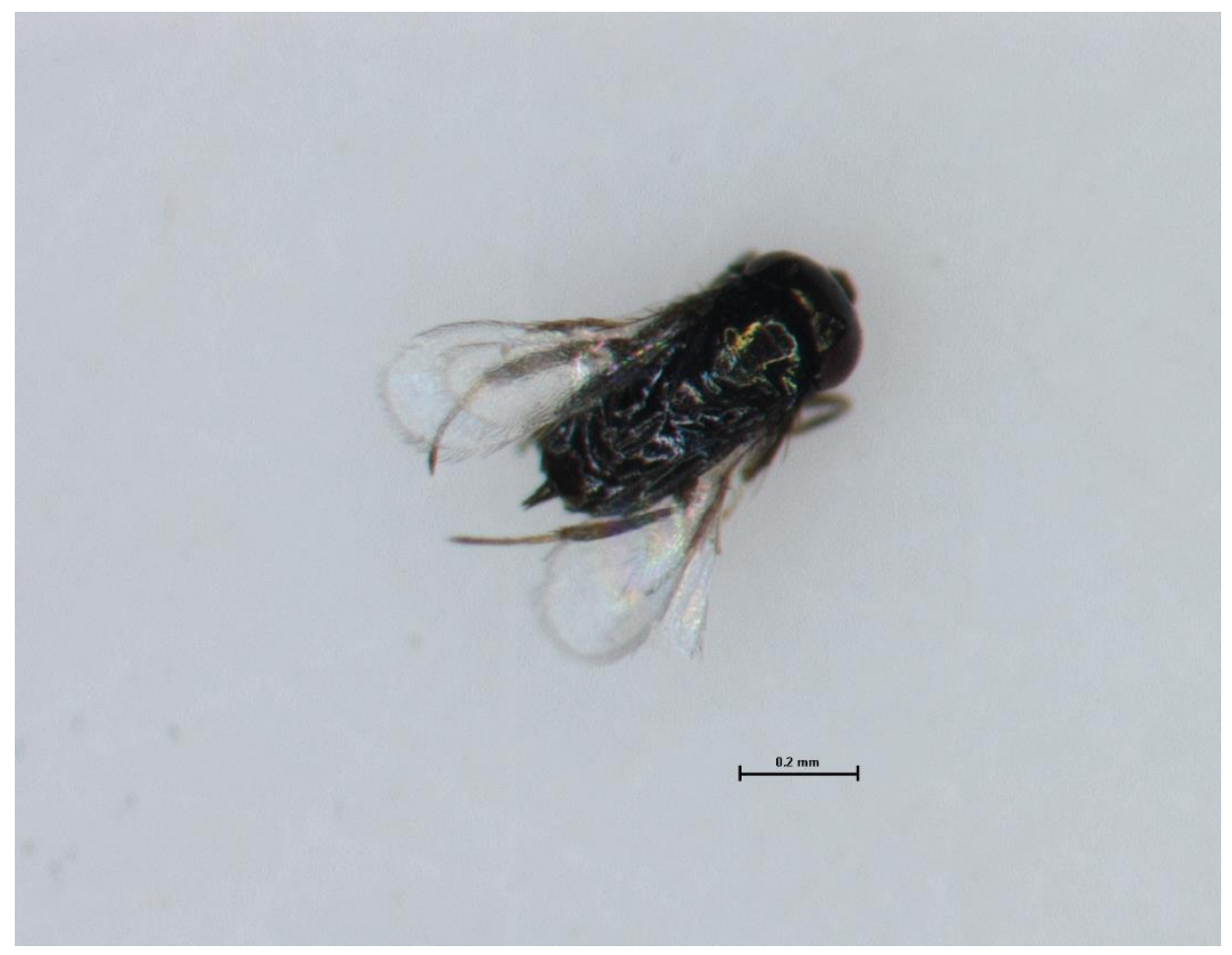

Figure 4 : Chartocerus hyalipennis. 


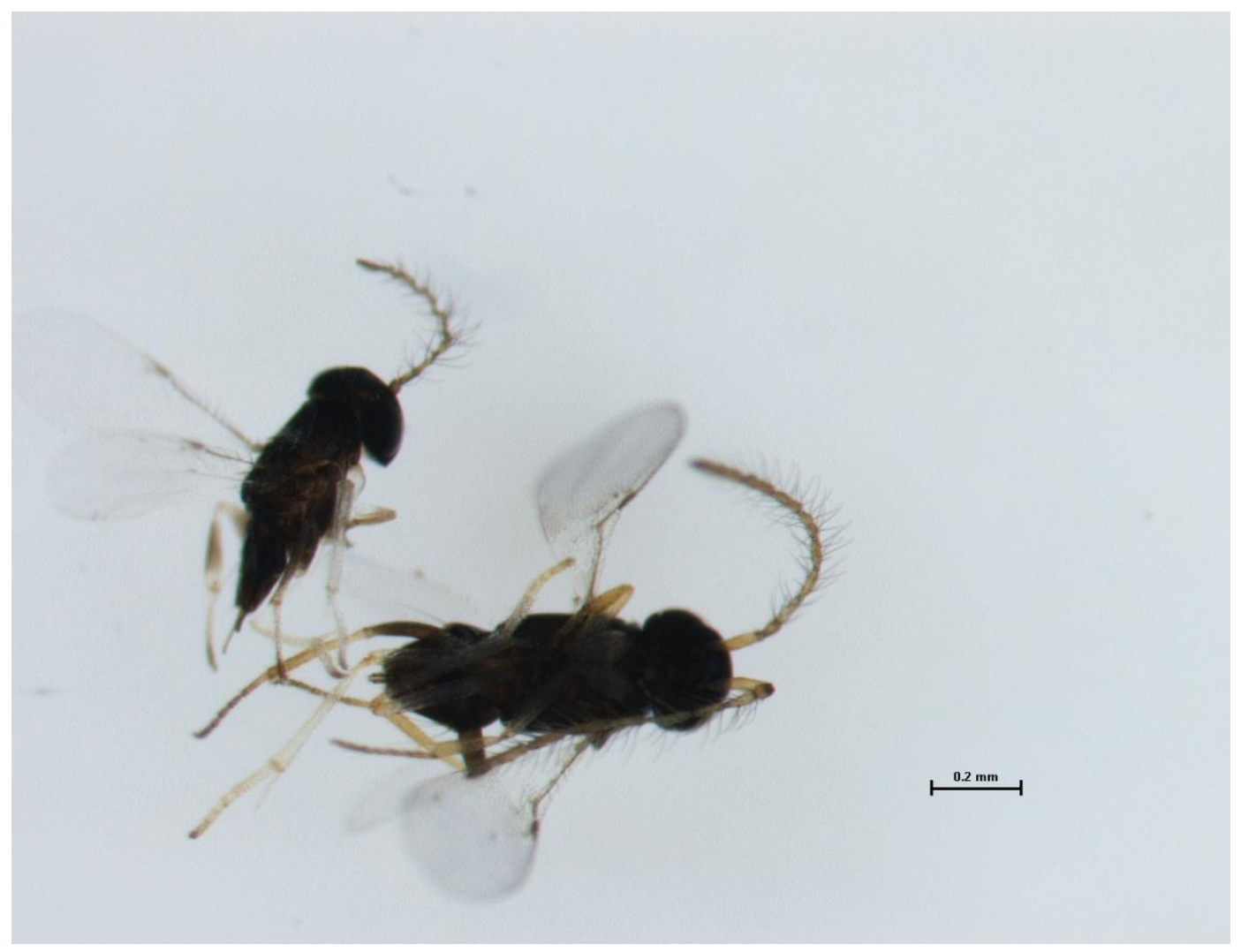

Figure 05 : Pachineuron $s p$

Tableau 3 : Taux d'hyperparasitisme au niveau de chaque site.

\begin{tabular}{lc}
\hline Sites & Taux d'hyperparasitisme (\%) \\
\hline Lycée Savorgnan de Brazza & 60 \\
\hline Jardin d'essai & 60 \\
\hline Kombé & 66,66 \\
\hline
\end{tabular}

\section{DISCUSSION}

Au terme de ce travail, la présence du parasitoïde E. lopezi en République du Congo a été confirmée (Biassangama et al., 1989; Neuenschwander 2001, 2004). Jusqu'à présent, E. lopezi était reconnu comme étant le seul parasitoïde primaire exotique de $P$. manihoti en Afrique tropicale et subtropicale (Nenon et al., 1985 ; Biassangama et al., 1989). Au cours de cette étude, un autre parasitoïde Acerophagus coccois (Smith) non encore décrit en
République du Congo a été identifié. Il est identifié comme étant un parasitoïde de Phenacoccus herreni, une autre cochenille qui sévit en Amérique du sud (Dorn et 2001, Bellotti et al., 1999 ; Bento et al., 1999) et qui peut également s'attaquer aux autres cochenilles telles que $P$. manihoti (Calatayud, 2011).

Le parasitoïde $A$. coccois peut avoir été introduit accidentellement avec des boutures de manioc qui sont constamment importées et 
expérimentées au centre de vulgarisation des techniques agricoles de Kombé dans le but d'améliorer les performances du manioc. Il est d'ailleurs, plus abondant dans ce site de Kombé que dans les deux autres. Entrainé par les vents et bénéficiant des conditions climatiques favorables (température, humidité relative) il se serait ainsi propagé. La disponibilité des ressources et l'absence probable des hyperparasites, pourraient justifier son acclimatation rapide et son expansion dans les autres sites. Cette présence présente un avantage dans la lutte contre le ravageur. En effet, l'action concertée des deux parasitoïdes et des ennemis naturels indigènes devrait jouer un rôle plus efficace pour contrôler $P$. manihoti.

Comparé à $E$. lopezi, le parasitoïde $A$. coccois est une espèce grégaire qui malgré un faible taux de production de descendants, a une stratégie qui consiste à déposer plusieurs œufs en même temps pour compenser son nombre faible de ponte (Dorn et al., 2001). Cette stratégie peut aussi justifier le nombre plus important d'individus de A. coccois rencontré dans les trois sites

La différence constatée selon les sites, du nombre de momies peut s'expliquer par une différence d'infestation par P. manihoti. En effet, en considérant que les populations des deux parasitoïdes $E$. lopezi et $A$. coccois sont stables et proportionnellement reparties, on peut admettre que le nombre de momies récoltées est proportionnel au degré d'infestation des sites par P. manihoti. Le taux d'émergence moyen à l'échelle des 3 sites est égal à 46.52\%, c'est-à-dire qu'il y a approximativement une momie sur deux d'où n'ont pas émergé des arthropodes. Fabre et Nenon (1997) expliquent cela par le fait que le processus d'émergence peut être affecté par la résistance de l'hôte, par une réaction de type immunitaire qui aboutit à l'emprisonnement de la larve dans une capsule mélanisée. Il intervient à des degrés divers selon les régions mais, il est admis que l'intensité de cette réaction immunitaire est amplifiée par les fortes températures.
La présence ou non des hyperparasites dans la «biocénose momie », est essentielle pour l'efficacité du parasitoïde E. lopezi. En effet, trente-six ans après l'introduction de ce dernier au Congo, les taux d'hyperparasitisme trouvés dans cette étude varient entre 60 et $66,66 \%$. Ils correspondent à ceux signalés par Biassangama et al. (1989). Les hyperparasites demeurent numériquement plus importants que le parasitoïde E. lopezi. Le rapport entre ce dernier et les hyperparasites est en faveur de ceux-ci. En 1987 Biassangama et Moussa avaient trouvé sept hyperparasites de E. lopezi, tandis que la présente étude n'en a révélé que trois. Malgré ce faible effectif, le problème de l'efficacité du parasitoïde E. lopezi dans la lutte contre la cochenille du manioc se pose toujours à cause des taux d'hyperparasitisme élevés. Les hyperparasites exercent une action dépressive importante sur la multiplication de $E$. lopezi et le rendent inefficace.

\section{Conclusion}

Les résultats $\mathrm{du}$ présent travail confirment bien la présence de E. lopezi parasitoïde de $P$. manihoti et des hyperparasites habituellement signalés dans de nombreux pays sous les tropiques ainsi qu'en République du Congo. Ce sont : Prochiloneurus insolitus, Chartocerus hyalipennis et Pachineuron sp. Ces hyperparasites pourraient avoir une action dépressive sur la dynamique du parasitoïde. $\mathrm{La}$ présence d'un deuxième parasitoïde, A. coccois est un élément nouveau et prometteur dans la lutte contre $P$. manihoti. Elle prouve aussi que l'entomofaune de la cochenille du manioc est dynamique au Congo. Cependant, des études suivies doivent être menées afin d'assurer un contrôle régulier de ses différentes composantes.

\section{REMERCIEMENTS}

Les auteurs remercient le proviseur du lycée Pierre Savorgnan de Brazza, les responsables du centre Agri Congo de Kombé et du jardin d'essai situé dans le campus I pour leur avoir donné les autorisations d'effectuer des prélèvements au sein de leurs exploitations. 


\section{REFERENCES}

Abderahim M-S, Diatte M, Labou B, SOW G, Diarra K. 2018. Inventaire et distribution des principaux arthropodes ravageurs du manioc (Manihot esculenta CRANTZ) au Tchad. Int. J. Biol. Chem. Sci., 12(6): 2589-2601.

Atachi P, Matchi B, Bachabi F, Yehouenou A, Rurema DG, 2011. Les entomophages de la Cochenille du Manioc Phenacoccus manihoti Matile-Ferrero, 1977 au Bénin : inventaire des différentes espèces. L'Entomologiste, 67(4): 227-233.

Bellotti AC, Smith L, Lapointe SL. 1999. Recent advances in cassava pest management. Annual Review of Entomology, 44: 343-370.

Ben-Dov Y, German V. 2003. A Systematic Catalogue of the Diaspididae (Armoured Scale Insects) of the World, Subfamilies Aspidiotinae, Comstockiellinae and Odonaspidinae. Intercept, Andover, 1111 pp.

Bento J, De Moraes G, Bellotti A, Castillo J, Warumby J, Lapointe S. 1999. Introduction of parasitoids for the control of the cassava mealybug Phenacoccus herreni (Hemiptera: Pseudococcidae) in north-eastern Brazil. Bulletin of Entomological Research, 89(5): 403-410.

Biassangama A, Moussa JB. 1987. Les parasites d'Epidinocarcis (Apoanagyrus) lopezi (Hymenoptera : Encyrtidae) au Congo. Agronomie Tropicale, 42: 301304.

Biassangama A. 1984. Etude du parasitisme des cochenilles pseudococcidae par les hyménoptères Encyrtidae. Application à la lutte biologique contre la cochenille du manioc Phenacoccus manihoti en République du Congo. Thèse de $3^{\text {ème }}$ cycle, université de Rennes1, 176 p.

Biassangama A, Le Rü B, Yziquel V, Kiyindou A, Bimangou AS. 1989. L'entomocénose inféodée à la cochenille du manioc, Phenacoccus manihoti (Homoptera : Pseudococcidae) au Congo, cinq ans après l'introduction d'Epidinocarsis lopezi (Hymenoptera : Encyrtidae).
Annales de la Société Entomologique de France, 25(3) : 315-320.

Boivin G. 2001. Parasitoïdes et lutte biologique : paradigme ou panacée ? VertigO - La revue électronique en sciences de l'environnement, 2 : 29-35.

Butt TM. 2002. Use of entomogenous fungi for the control of insect pests. In The Mycota Agricultural Applications, Kempken F (ed.). Springer: Berlin; 111-134.

Calatayud PA. 2011. Interactions plantesinsectes. Habilitation à Diriger des Recherches (HDR)-IRD (UR 072), CNRS (UPR 9034), Université Paris Sud 11. Av. de la Terrasse, Bât. 13, 91198 Gifsur Yvette Cedex, 86 p.

Daouda D. 2018. Le manioc ivoirien : origine et expansion dans le reste du monde, du 16ème au 20ème siècle. European Journal of Social Sciences Studies, 2(10).

Dorn B, Mattiacci L, Bellotti AC, Dorn S. 2001. Host specificity and comparative foraging behaviour of Aenasius vexans and Acerophagus coccois, two endoparasitoids of the cassava mealybug. Entomologia Experimentalis et Applicata, 99(3): 331-339.

Fabres G, Le Rü B. 1988. Etude des relations Plante-insectes pour la mise au point de méthodes de régulation des populations de la cochenille du manioc. 7th Symp. of the Int. Soc. Root Crops., Pointe-à-Pitre, Guadeloupe, 563-577

Fabres G, Nénon JP. 1997. Biodiversité et lutte biologique : le cas de cochenille du manioc en Afrique. Journal of African Zoology, 111(1) : 7-15.

Herren HR, Lema KM. 1982. CMB - premières sorties réussies. Bureaux agricoles du Commonwealth, Biocontrol News and Information, 3, p 185.

IITA 2000. Lutte contre les ravageurs du manioc. Guide de la pratique de lutte intégrée à l'usage des vulgarisateurs. Cotonou, République du Bénin, 36p.

Iziquel Y, Le Rü B. 1989. Influence de l'hyperparasitisme sur les populations de l'encyrtide Epidinocarsis lopezi, un parasitoïde de Phenacoccus manihoti introduit au Congo. Entomologia 
Experimentalis et Applicata, 52(3) : 239247.

Lakhdari W, Doumandji Mitich B, Dahliz A, Doumandji S, Bouchikh Y, M'lik R, Soud A, Hammi H. 2015. Essai de lutte biologique contre Brachytrupes megacephalus, lefebvre, 1827 (orthoptera, gryllinae) par l'utilisation des champignons entomopathogenes. Revue des BioRessources, 5(1) : 37-49.

Le Rü B. 1989. Lutte biologique contre les ravageurs. In : quarante ans de recherches scientifiques au Congo sur « les hommes et l'environnement $»$. Brazzaville, le 23 novembre 1989, OROSTOMMESSCRE, p38-40.

Lema KM, Herren HR. 1985. Release and establishment in Nigeria of Epidinocarsis lopezi, a parasitoid of the cassava mealybug, Phenacoccus manihoti. Entomologia Experimentalis et Applicata, 38(2) : 171-175.

Nenon JP, Fabres G, Biassangama A. 1985. Epidinocarcis lopezi (hym. Encyrtidae), parasitoide introduit au Congo pour la regulation des populations de la cochenille du manioc Phenacoccus manihoti (hom : Pseudococcidae). In: 7th international symposium of the society for tropical Root Crops (ISTRC), Pointe-à-pitre, Guadeloupe, 2 p.

Neuenschwander P. 2001. Biological Control of the Cassava Mealybug in Africa: A Review. Biological Control, 21: 214-229.
Neuenschwander P. 2004. Harnessing nature in Africa. Nature, 432: 801-802.

Obame Minko D, Békon AK. 2005. Etude de l'entomofaune associée à la cochenille du manioc Phenacoccus manihoti MatileFerrero, en Côte d'Ivoire. Tropicultura, 23(3) : 136-140.

Odebiyi, Bokonon-Ganta AH. 1986. Biologie de l'Epidinocarsis (Apoanagyrus) lopezi (Hymenoptera : Encyrtidae), un parasite exotique de la cochenille du manioc, Phenacoccus manihoti (Homoptera : Pseudococcidae) au Nigeria. Entomophaga, 31 (3) : 251-260.

Pynaert L. 1951. Le manioc. Collection technique du ministère de la coopération du royaume de Belgique, 2è édition, Bruxelles, $166 \mathrm{p}$.

Réseau National de Télécommunications, l'Enseignement et la Recherche (RENATER). https://www.renater.fr/

Silvestre P. 1973. Aspects agronomiques de la production du manioc à la ferme d'Etat de Mantsoumba. - IRAT, rapport de mission. Inst. Rech. Agro. Trop., Paris, 35 pages

Yonow T, Kriticos Darren J, Ota N. 2017. The potential distribution of cassava mealybug (Phenacoccus manihoti), a threat to food security for the poor. PLoS ONE, 12(3). 